This department welcomes short notes and problems believed to be new. Contributors should include solutions where known, or background material in case the problem is unsolved. Send all communications concerning this department to I. G. Connell, Department of Mathematics, McGill Universit: Montreal, P.Q.

\title{
GENERALIZATION OF A BRACKET FUNCTION FORMULA OF L. MOSER
}

H. W. Gould

In Problem P 60 L. Moser has proposed the formula

(1) $\sum_{k=1}^{n}[\sqrt{k}]=[\sqrt{n}] \frac{6 n-2[\sqrt{n}]^{2}-3[\sqrt{n}]+5}{6}$

This can be expressed in the more elegant form

$$
\sum_{k=1}^{n}[\sqrt{k}]=m(n+1)-\frac{m(m+1)(2 m+1)}{6}, m=[\sqrt{n}] .
$$

The formula has been given in the literature a number of times. For example, it was proved by Bouniakovsky [1].

For cube roots we have

$$
\sum_{k=1}^{n}[\sqrt[3]{k}]=m(n+1)-\frac{m^{2}(m+1)^{2}}{4}, m=[\sqrt[3]{n}] .
$$

We may generalize these in the single formula

(4) $\quad \sum[\sqrt[a]{\sqrt[a]{k}}[\sqrt[a]{n}]$

$$
\sum_{k=1}^{n}[\sqrt[a]{k}]+\sum_{k=1}^{[\sqrt[a]{n}]}
$$


This may be easily found from the general theorem for arrays of numbers

$$
\sum_{k=1}^{n} \sum_{j=1}^{[\sqrt[a]{k}]} A_{j, k}=\sum_{j=1}^{[\sqrt[a]{n}]} \underset{k=j}{\sum_{k}} A_{j, k},
$$

which is readily proved by induction. Relation (4) follows when $A_{j, k}=1$.

A slight generalization of (4) was given by Zeller [3] .

For another example of the general relation (5) choose $A_{j, k}=2 j-1$ and recall that $\sum_{j=1}^{n}(2 j-1)=n^{2}$. We find

$$
\sum_{k=1}^{n}[\sqrt[a]{k}]^{2}=\sum_{j=1}^{[\sqrt[a]{n}]}(2 j-1)\left(n-j^{a}+1\right)
$$

which we may rewrite in the form

$$
\sum_{k=1}^{n}[\sqrt[a]{k}]^{2}=(n+1) m^{2}-2 \sum_{j=1}^{m} j^{a+1}+\sum_{j=1}^{m} j^{a}, m=[\sqrt[a]{n}] .
$$

If we set $a=2$ in this we find after a little algebra a nice companion to Moser's relation (in the form (2))

$$
\sum_{k=1}^{n}[\sqrt{k}]^{2}=m^{2}(n+1)-\frac{m(m+1)\left(3 m^{2}+5 m+1\right)}{6} ; m=[\sqrt{n}] \text {. }
$$

There are many relations similar to (5) which are not too well-known and which may be used to sum series involving the bracket function. We shall mention two others here:

$$
\sum_{k=a}^{n} \sum_{j=a}^{[k / r]} A_{j, k}=\sum_{j=a}^{[n / r]} \underset{k=r j}{\sum} A_{j, k},
$$


(10) $\sum_{k=1}^{n} \sum_{j=1}^{2^{k}-1} A_{j, k}=\sum_{j=1}^{2_{k=1}^{n}-1} \sum_{\left[\log _{2} j\right]}^{n} A_{j, k}$

Inversion of order of summation quite naturally brings in to play the inverse of the function which is used for the upper limit of summation on the left.

\section{REFERENCES}

1. V. Bouniakovsky, Démonstration de quelques propositions relatives à la fonction numérique $\mathrm{E}(\mathrm{x})$, Art. 3 ème

Pétersbourg, Mélanges, p. 169-201. Cf. Jahrbuch đber die Fortschritte der Mathematik, v. 16(1884), 150-151.

2. L. Moser, Problem P 60, Canadian Math. Bulletin, 5(1962), 310.

3. Chr. Zeller, Über Summen von grössten Ganzen bei arithmetischen Reihen, G8tt, Nachr. 1879, 243-268.

West Virginia University

Editor's comment: Formulas (5), (9), and (10) are examples of the following general identity. Let $f(k), k=1,2, \ldots$, be a non-decreasing sequence of positive integers and define, following Lambek and Moser (Amer. Math. Monthly, 1954, p. 454), the 'inverse' by

$$
f *(n)=\text { number of } m \text { such that } f(m)<n \text {, }
$$

(so that $f * *=f$ ). For example if $f(n)=[\sqrt[a]{n}]$, then $f *(n)=n^{a}-1$. We have 


$$
\sum_{k=1}^{n} \sum_{j=1}^{f(k)} A_{j k}=\sum_{j=1} \sum_{k=f *(j)+1}^{f} A_{j k}
$$

(in one case we are summing first by rows then by columns, in the other case in the opposite order). Lambek and Moser give several other entertaining examoles; e.g. ,

$$
\begin{aligned}
& f(n)=p_{n}, \text { the } n^{\text {th }} \text { prime, } \\
& f * k(n)=\pi(n-1), \text { the number of primes }<n .
\end{aligned}
$$

Kimura, in his solution of problem $\mathrm{P} 60$, points out that since

$$
\begin{aligned}
& (1-x) \sum_{k=1}^{\infty}[\sqrt[r]{k}] x^{k}=\Sigma_{k=1}^{\infty} x^{k^{r}}=g(x) \text {, say, we have } \\
& \sum_{k=1}^{n}[\sqrt[r]{k}]=\frac{1}{2 \pi i} \int_{C} \frac{g(z)}{z^{n+1}(1-z)^{2}} d z
\end{aligned}
$$

where $C$ is a contour round the origin, contained in the unit circle.

The analogue in the general case is

$$
(1-x) \sum_{n=1}^{\infty} f(n) x^{n}=x \sum_{n=1}^{\infty} x^{f *(n)}
$$

(note that some of the exponents $f *(n)$ may be equal); and if $f(n)$ does not increase too rapidly, so that the series have positive radii of convergence, there is a similar contour integral formila for $\sum_{k=1}^{n} f(k)$. 